\title{
Prise en charge psycho-sexologique du patient impuissant
}

\author{
Robert GELLMAN \\ Ecole française de Sexologie, 3 rue Copernic, 75116 PARIS
}

Cette communication a été présentée à la 7ème Journée de l'Ecole Française de Sexologie, Paris, 24 Septembre 1994.

\section{RESUME}

Le symptôme impuissance est dans l'immense majorité des cas surdéterminé.

En ce qui concerne l'abord thérapeutique il existe des stratégies diverses : andrologique, analytique, psychocomportementale, ...

Le progrès de ces dernières années a été la mise en place de modèles intégrés :

- le modèle américain d'Helen Kaplan,

- le modèle genevois de Willy Pasini,

- le modèle parisien que nous proposons se nomme sexothérapie analy. tique et associe plusieurs étapes nettement différenciées.

La prise en charge de l'impuissance ne doit pas se limiter à un traitement uniquement pharmacologique.

Mots clés : impuissance, stratégie, modèles intégrés.

\section{INTRODUCTION}

\section{La surdétermination des facteurs.}

L'impuissance érectile est la résultante d'un certain nombre de facteurs :
- Les facteurs organiques sont bien connus et se décomposent en facteurs vasculaires, neurologiques, hormonaux...

- Les facteurs psychogènes sont également multiples.

Nous différencions quant à nous :

Les facteurs psychologiques intra-psychiques. Il peut s'agir de la réaction à des stress : deuils, séparations, révélation d'un secret (relation extra-conjugale d'un des patient, problèmes de jalousie, problèmes d'inceste, ...)

Il peut s'agir de décompensation névrotique, ou encore d'homosexualité latente empêchant le sujet d'investir sa partenaire comme objet d'amour.

Ces facteurs psychogènes intra-psychiques sont à différencier des facteurs conjugaux liés à un certain nombre des conflits : conflits de pouvoir, conflits liés aux familles respectives, conflits liés aux enfants, dûs aux problèmes d'éducation ou de scolarité.

Nous faisons une place à part aux problèmes relationnels, au sens large du terme, que ce soient les difficultés relationnelles avec le milieu professionnel, les difficultés avec la famille ou bien les frustrations rencontrées dans les relations amicales.

Les facteurs sociologiques, au sens large du terme se doivent d'être analysés, que ce soient les problèmes de chômage, de la retraite, ou bien les situations liées à la pro- 
miscuité (logements trop petits, trop bruyants...) avec un environnement peu favorable aux échanges affectifs, à l'intimité.

Il peut encore s'agir des difficultés pécuniaires ou bien de problèmes liés à la transplantation.

Au niveau psychologique il est essentiel de comprendre la nature exacte des difficultés du patient, de même qu'il est essentiel de différencier la part des facteurs vasculaires artériels ou veineux, des facteurs neurologiques ou hormonaux.

Nous avons schématisé l'interaction de ces différents facteurs par une formule (Tableau 1):
On remarquera que ces facteurs ne s'additionnent pas, mais se multiplient, ce qui, en accroît considérablement l'impact.

En général la présence d'un seul facteur ne suffit pas à déclencher le symptôme. Un deuxième ou un troisième facteur entraînera par contre l'apparition de difficultés érectiles.

\section{LES STRATEGIES THERAPEUTIQUES}

La première en date est la stratégie analytique (Tableau 2) qui s'applique lorsque les facteurs organiques ont été écartés.

Elle repose sur les découvertes de S. Freud concernant l'inconscient. La technique analytique s'appuie sur la libre association,

Tableau 1 : Surdétermination des facteurs psychogènes.

$$
O \times P \times Q \times R \times S=\text { T.S. }
$$

$\mathrm{O}=$ ORGANIQUES

$\mathrm{P} \quad=\quad$ PSYCHIQUES

- réactionnels : deuil, séparation, révélation d'un secret

- décompensation névrotique

- homosexualité latente

- etc.

$\mathrm{Q}=$ CONJUGAUX

- conflits de pouvoir

- conflits liés aux familles respectives

- conflits liés aux enfants (éducation, scolarité, ...)

- etc.

$\mathrm{R}=$ RELATIONNELS au sens large

- difficultés relationnelles avec le milieu professionnel

- difficultés relationnelles avec la famille

- difficultés relationnelles avec les amis

$\mathrm{S}=$ SOCIOLOGIQUES

- chômage

- retraite

- promiscuité (logements trop petits, trop bruyant, ...)

- difficultés pécuniaires

- transplantation

- etc.

T.S. $=$ TROUBLES SEXUELS 
$->$ FREUD

- Libre Association

- Analyse des rêves

- Analyse des résistances

- Analyse du transfert

$\rightarrow$ HYPOTHESE

La résolution des conflits inconscients entraîne l'établissement d'une sexualité normale

$\rightarrow$ W. REICH

- Lecture du corps

- Libération de l'énergie bloquée par

l'utilisation d'exercices corporels appropriés

l'analyse des rêves, l'analyse des résistances et l'analyse du transfert.

La mise en évidence des causes profondes des conflits, le "sens" donné au symptôme doivent dans l'hypothèse freudienne entraîner le rétablissement d'une sexualité normale.

W. REICH pour sa part se livre à une lecture $\mathrm{du}$ corps, qui amène à une compréhension des conflits vécus par le sujet. Dans un second temps l'utilisation d'exercices corporels appropriés va entraîner une libération de "l'énergie bloquée" au niveau d'un certain nombre de segments corporels. Le sujet va pouvoir accéder à une libération énergétique et vivre pleinement son orgasme.

A travers les travaux d'Alexander Lowen concernant la bioénergie, les hypothèses de W. REICH vont avoir une diffusion très importante aux U.S.A.

\section{LA STRATEGIE DITE "ANDROLOGIQUE"}

Est celle pratiquée par nos collègues urologues et par les médecins généralistes non formés aux techniques psycho-sexologiques (Tableau 3).

A la suite d'un bilan complémentaire comprenant des investigations biologiques, hor-

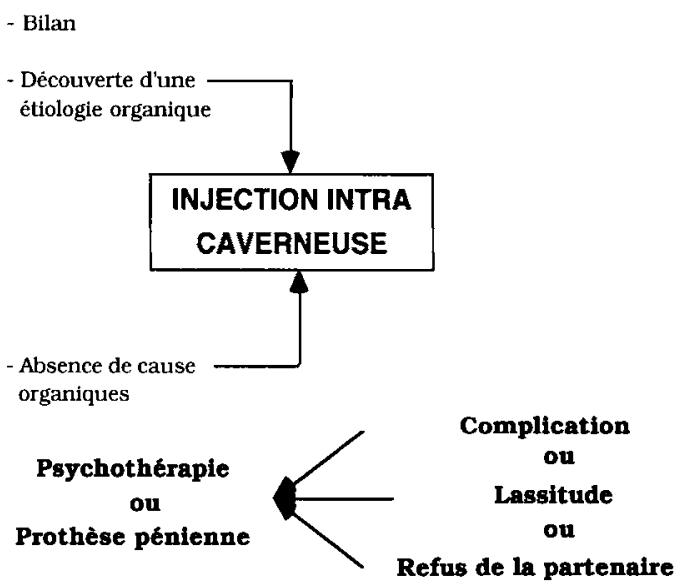

monales, vasculaires, neurologiques, un ou plusieurs facteurs organiques sont découverts.

On propose alors des traitements palliatifs, per os ou voie intra-musculaire (traitements hormonaux par exemple) ou injections de drogues vaso-actives par voie intra-caverneuse.

Il est à noter qu'en l'absence de facteurs organiques les injections intra-caverneuses sont également proposées, en espérant que le patient en récupérant une confiance dans ses capacités érectiles sera définitivement guéri de son impuissance.

En cas de complications dues aux injections intra-caverneuses ou en cas de lassitude dues aux contraintes du traitement, en cas de refus de la partenaire, le patient est adressé au psycho-sexologue, au psychanalyste ou bien il lui est proposé la pose d'une prothèse pénienne.

\section{LA STRATEGIE PSYCHO-COMPORTEMENTALE}

La stratégie psycho-comportementale (Tableau 4) part d'hypothèses conceptuelles très différentes des concepts analytiques freudiens.

Ces techniques comprennent diverses étapes (approche de la partenaire, dénuda- 
Tableau 4: Stratégie psycho-comportementale.

- Fractionnement du but à atteindre

- Remodelage de l'anticipation

- Restauration du scénario du comportement sexuel

- Correction et dédramatisation du discours interne

- Utilisation éventuelle dans le contexte d'injections intra caverneuses associées

Tableau 5 : Sexothérapie analytique. tion, préliminaires érotiques...), le patient est invité à remodeler de façon positive les différentes étapes qui conduisent au but à atteindre, il est encouragé à restaurer un scénario de comportement sexuel conduisant à la réussite et non à l'échec.

Parallèlement son discours interne est corrigé et dédramatisé.

C'est dans ce contexte de réassurance et de dédramatisation, de vision optimiste et positive que les injections intra-caverneuses sont proposées.

La stratégie que nous proposons est dénommée sexothérapie analytique (Tableau 5) et s'applique en cas d'absence de lésion organique prédominante. Elle comporte plusieurs étapes :

- La première étape est une thérapie analytique courte, c'est à dire inférieure à

EN L'ABSENCE DE LESION ORGANIQUE

A

PSYCHOTHERAPIE

ANALYTIgUE COURTE

$<20$ séances

centrée sur le symptôme

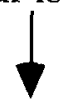

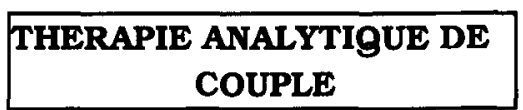

centrée sur

l'analyse des conflits relationnels

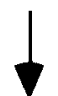

B/

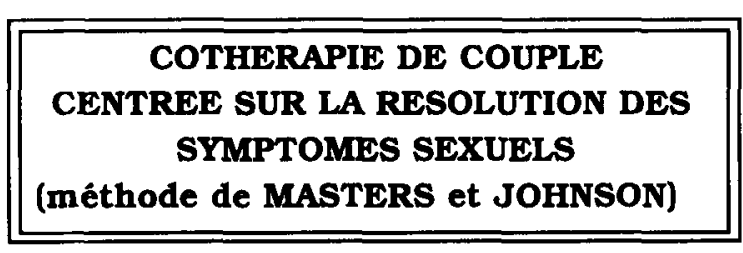

C/ En cas de difficultés dans l'évolution de la thérapie de couple.

\section{Entretiens individuels parallèles}

- Relaxation

- Fantasmothérapie 
vingt séances, centrée sur l'analyse du symptôme. Dans ce court laps de temps les phénomènes transférentiels ne sont en général pas analysés.

Ce peut être également une thérapie analytique de couple, où les deux partenaires consultent simultanément. Cette thérapie de couple est centrée sur l'analyse des conflits relationnels.

Cette première étape peut se conclure par une résolution du symptôme d'impuissance dans un nombre non négligeable de cas.

Il faut noter que dans $30 \%$ des cas d'impuissance le symptôme se résoud quel que soit le mode d'intervention proposé, que celui-ci soit pharmacologique, psychothérapique, relationnel ou corporel.

Les résultats de cette première étape du traitement sont conformes à cette donnée générale.

- Les patients ont été dès le départ informés de l'éventualité d'une seconde étape, avec cette fois ci, de façon obligatoire, la participation de leur partenaire. Il s'agit de la mise en place d'une cothérapie de couple, centrée sur la résolution du symptôme sexuel. La classique méthode de Masters et Johnson nécessitant l'intervention de deux cothérapeutes de sexe et de formation complémentaire. Les différentes étapes de cette thérapie doivent être menées avec rigueur.

L'hypothèse de Masters et Johnson qui se vérifie à chaque prise en charge, est que dans un couple ayant une certaine ancienneté un symptôme ne perdure que si les deux partenaires présentent chacun des difficultés.

C'est ainsi qu'en plus du symptôme d'impuissance nous aurons à traiter un problème d'absence de libido, de dyspareunie, d'anorgasmie coittale ou de frigidité chez la partenaire.

Une formation sexologique très sérieuse s'impose donc aux deux thérapeutes s'ils veulent traiter des patients en cothérapie.
Si cette condition est remplie, et que les deux patients sont motivés, la résolution du symptôme ne pose en fait que des problèmes techniques relativement mineurs.

Dans quelques cas rarissimes, en cas de blocage à l'une des étapes de la cothérapie, on peut mettre en place parallèlement : des entretiens individuels très limités dans le temps, une relaxation ou un travail sur les fantasmes originaires (fantasmothérapie analytique).

\section{CONCLUSION}

Le symptôme impuissance est dans l'immense majorité des cas surdéterminé, il associe à des facteurs organiques éventuels, des facteurs psychologiques, relationnels, conjugaux, sociaux.

C'est la présence de plusieurs facteurs de risque qui va déterminer l'apparition du symptôme, car ceux-ci ne s'additionnent pas mais se multiplient.

Les stratégies de traitement actuellement proposées sont diverses :

- stratégie andrologique,

- stratégie analytique,

- stratégie psycho-comportementale,

- la sexothérapie analytique.

La mise en place des grands modèles thérapeutiques (analytique, mastérien, andrologique...) a constitué une étape importante à laquelle est venue s'ajouter la mise en place des modèles intégrés.

Parmi ces modèles intégrés nous citerons :

- le modèle américain (côte est des U.S.A.) d'Helen KaPLAN associe l'interprétation analytique à la démarche pédagogique de Masters et Johnson ;

- le modèle genevois de W. PAsini associe un travail analytique parallèle à un abord corporel ;

- le modèle parisien que nous proposons se nomme sexothérapie analytique. Il associe un travail analytique court, cen- 
tré sur le symptôme suivi d'une cothérapie de couple. On utilise, si besoin, parallèlement, un travail de relaxation ou un travail sur les fantasmes(fantasmothérapie analytique) pour lever des résistances éventuelles au bon déroulement de la cothérapie de couple.

La mise en place des modèles intégrés associant divers modes d'abord, ménageant des étapes, constitue un progrès supplémentaire, permettant la résolution des difficultés rencontrées.

\section{ABSTRACT}

Psycho-sexual management of the impotent patient

Robert GeLLMaN

The symptom of impotence is, in the vast majority of cases, extremely well- defined. However, therapeutic approaches incorporate various strategies, including : andrological, analytical and behavioural.

A great advance in recent years was due to the development of "integrative models" such as the "American Model" by Helen Kaplan, the "Geneva Model" of Willy Pasini, and our proposed "Parisian Model", which is founded on the principles of analytical sexotherapy, and comprises several clearly defined stages. The management of impotence must not be restricted only to pharmacological treatment.

Key works : impotence, strategies, integrated models, therapy. 